\title{
On Schappert's characterization of strictly unimodal plane curve singularities
}

\author{
Yuri A. Drozd * \\ Faculty of Mechanics and Mathematics \\ Kyiv Taras Shevchenko University \\ $252033 \mathrm{Kyiv}$ \\ UKRAINE \\ Gert-Martin Greuel \\ Universität Kaiserslautern \\ Fachbereich Mathematik \\ Erwin-Schrödinger-Straße \\ 67663 Kaiserslautern \\ GERMANY
}

\section{Introduction}

The representation theory of curve singularities (more precisely, of their local rings) has turned out to be closely related to their deformation properties. Namely, as was shown in [6],[9],[7], such a ring $\mathrm{R}$ is of finite type, that is has only finitely many torsion-free indecomposable modules (up to isomorphism), if and only if it dominates one of the so called simple plane curve singularities in the sense of [1]. In [4] the authors have shown that $\mathrm{R}$ is of tame type, that is it has essentially only 1-parameter families of indecomposable torsionfree modules, if and only if it dominates one of the unimodal plane curve singularities of type $\mathrm{T}_{p q}\left(\mathrm{~T}_{p q 2}\right.$ in the classification of [1]).

These singularities form the "serial" part of the list of all unimodal plane curve singularities. There are also 14 "exceptional" ones, which happen to be wild, that is they possess $n$-parameter families of (non-isomorphic) indecomposable modules for arbitrary large $n$. The bimodal plane curve singularities in the sense of [1] are also wild. Nevertheless, in [11] was shown that all uni- and bimodal plane curve singularities possess only 1-parameter families of ideals.

\footnotetext{
* Supported by DFG and International Science Foundation, grant RKJ000.
} 
In [13] these singularities are called strictly unimodal and we prefer to use this terminology.

The aim of our paper is to show that the strictly unimodal plane curve singularities are in some sense "universal" among those having not more than 1-parameter families of ideals. Namely, we prove that a curve singularity has this property if and only if it dominates one of the strictly unimodal plane curve singularities. Moreover, we prove this result for curve singularities over an algebraically closed field of arbitrary characteristic. For this we use, instead of the definition of such singularities by the corresponding equations, their characterization via parametrization given in [11]. Note that it follows from $[6],[9],[7]$ that a curve singularity has only finitely many non-isomorphic ideals if and only if it is of finite type (in contrast with the case of 1-parameter families). We use the parametric characterization as a definition in positive characteristic (with special care in characteristic 2) and call them ideal-unimodal in view of the main theorem of this paper.

The proof of this theorem follows the same scheme as that of the main result on tameness from [4]. Namely, we first introduce some "overring conditions" for the ring $\mathrm{R}$ and show that whenever they do not hold, R possess 2-parameter families of non-isomorphic ideals. Then we show that these conditions imply that $\mathrm{R}$ dominates a strictly unimodal plane curve singularity. To accomplish the proof, we need also to show that any strictly unimodal plane curve singularity has not more than 1-parameter families of ideals. But indeed, one can calculate all ideals of these rings. This has already been done in [11] and [12]. Although Schappert used the "definition via equations", one can verify (and we do it here for three most complicated examples) that his calculations depend only on the parametrization of these rings. This calculation of ideals shows that all strictly unimodal plane curve singularities really have only 1-parameter families of ideals. Moreover, using the parametrization, we can extend this result to curve singularities over algebraically closed fields of positive characteristic, that is, to ideal-unimodal singularities.

\section{Preliminaries}

Notation 1.1 Throughout this article we use the following notations:

- $\mathrm{R}$ denotes a complete local noetherian ring without nilpotent elements.

- $\mathrm{Q}$ its full ring of fractions.

- $\mathfrak{m}=\operatorname{rad} \mathrm{R}$ its unique maximal ideal.

- $\mathbf{k}=\mathrm{R} / \mathfrak{m}$, the residue field of $\mathrm{R}$.

- $\mathrm{R}_{0}$ its normalization, i.e. its integral closure in $\mathrm{Q}$.

- $\mathrm{R}_{i}=\mathfrak{m}^{i} \mathrm{R}_{0}+\mathrm{R}$ (a local ring for $i>0$ ). 
- $\mathfrak{m}_{i}=\mathfrak{m}^{i} \mathrm{R}_{0}+\mathfrak{m}$ (the maximal ideal of $R_{i}$ for $i>0$ ).

- $\mathrm{d}(M)=\operatorname{dim}_{\mathbf{k}}(M / \mathfrak{m} M)$, the minimal number of generators of an $\mathrm{R}$ module $M$.

- $\mathrm{d}_{i}=\mathrm{d}\left(\mathrm{R}_{i}\right)$.

Later on we suppose $\mathbf{k}$ to be algebraically closed.

Definition 1.2 $\mathrm{R}$ is said to be a curve singularity provided it satisfies the following conditions:

1. $R$ is a $\mathbf{k}$-algebra and $R / \mathfrak{m}=\mathbf{k}$.

2. $\mathrm{R}$ is of Krull dimension 1 .

Such rings are just the completions of the local rings of points of reduced algebraic curves over the field $\mathbf{k}$.

It is known that, in this case, $\mathrm{d}_{0}$ is finite and, moreover, $\mathrm{d}(I) \leq \mathrm{d}_{0}$ for each R-ideal $I$ (cf. [3]).

Recall the definitions related to families of R-modules (cf. [5],[10]). We shall consider here only full R-ideals, i.e. ideals $I$, such that $\mathrm{Q} I=\mathrm{Q}$ (later we omit the epithet "full").

Definition 1.3 Let $X$ be an algebraic variety over $\mathbf{k}$ and $\mathcal{I}$ an $\mathrm{R} \otimes \mathcal{O}_{X}$-idealsheaf, such that $\mathrm{Q} \mathcal{I}=\mathrm{Q} \otimes \mathcal{O}_{X}$ (the tensor product is over $\mathbf{k}$ ). Call $\mathcal{I}$ a family of ideals with base $X$ if it is flat over $\mathcal{O}_{X}$ and if $\mathcal{I} / r \mathcal{I}$ is $\mathcal{O}_{X}$-flat for each non-zero divisor $r \in \mathrm{R}$.

A series of such families, which are in some sense universal, can be constructed as follows. Consider the subvariety $\mathrm{B}(d)$ of the Grassmannian $\operatorname{Gr}\left(d, \mathrm{R}_{0} / \mathrm{R}\right)$, consisting of those subspaces, of $\mathrm{R}_{0} / \mathrm{R}$ of codimension $d$, which are $\mathrm{R}$ submodules in $\mathrm{R}_{0} / \mathrm{R}$. The pre-image $\mathcal{I}(d)$ in $\mathrm{R}_{0} \otimes \mathcal{O}_{\mathrm{B}(d)}$ of the canonical locally free sheaf of corank $d$ on $\mathrm{B}(d)$ is then a family of R-ideals and any other family can be "glued" from the inverse images of the families $\mathcal{I}(d)$ (cf. [5], Proposition 3.5 and Corollary 3.6). Hence, we are able to define, following [10], the number of parameters for $\mathrm{R}-i d e a l s$, $\operatorname{par}(1, \mathrm{R})$.

Definition 1.4 Let $\mathrm{B}(d, i)$ the subset of $\mathrm{B}(d)$ consisting of points $x$ such that the set $\{y \in \mathrm{B}(d) \mid \mathcal{I}(d)(y) \simeq \mathcal{I}(d)(x)\}$ (which is locally closed) has dimension $i$ and define

$$
\operatorname{par}(1, \mathrm{R})=\max _{d, i}\{\operatorname{dim} \mathrm{B}(d, i)-i\} .
$$

Note that $\mathrm{B}(d, i)$ is also locally closed in $\mathrm{B}(d)$ and that, intuitively, $\operatorname{par}(1, \mathrm{R})$ is the maximal number of independent parameters of isomorphism classes of $R$ ideals ( $=$ torsion free $R$-modules of rank 1 ). Hence, $\operatorname{par}(1, \mathrm{R})$ may be considered as the maximal dimension of a component of a moduli space parametrizing nonisomorphic $R$-ideals. 
We say that a ring $\mathrm{R}^{\prime}$ dominates $\mathrm{R}$ if $\mathrm{R} \subseteq \mathrm{R}^{\prime} \subseteq \mathrm{R}_{0}$. In this case, evidently, $\operatorname{par}\left(1, \mathrm{R}^{\prime}\right) \leq \operatorname{par}(1, \mathrm{R})$. It follows from [7], [3] that $\operatorname{par}(1, \mathrm{R})=0$ if and only if $\mathrm{R}$ dominates one of the so-called simple (or 0-modal) plane curve singularities in the sense of [1] (cf. also [13], [8]). We are going to prove an analogous fact concerning the strictly unimodal plane curve singularities (cf. [1], [13]) ${ }^{1}$ and extend this to arbitrary characteristic.

As $\mathbf{k}$ is algebraically closed, we may suppose that $\mathrm{R}_{0}=\prod_{i=1}^{s} \mathrm{D}_{i}$, where $\mathrm{D}_{i}=\mathbf{k}\left[\left[t_{i}\right]\right]$ (formal power series rings). The number $s$ is called the number of branches of $\mathrm{R}$. Let $t=\left(t_{1}, t_{2}, \ldots, t_{s}\right)$ and $v_{i}$ be the standard valuation in $\mathrm{D}_{i}$. For any element $r=\left(r_{1}, r_{2}, \ldots, r_{s}\right) \in \mathrm{R}_{0}$ define its (multi-)valuation as the vector $\mathbf{v}(r)=\left(v_{1}\left(r_{1}\right), v_{2}\left(r_{2}\right), \ldots, v_{s}\left(r_{s}\right)\right)$. In Table 1 we prefer to present the plane curve singularities in a parametrized form, that is given by their generators $x, y$ as complete subalgebra of $\mathrm{R}_{0}$. Such a presentation has the advantage that it is almost independent of the characteristic - only char $\mathbf{k}=2$ needs extra conditions. In the table the valuations $\mathbf{v}(x)$ and $\mathbf{v}(y)$ are given.

In view of Theorem 2.1 we propose the following definition:

Definition 1.5 A plane curve singularity with complete local ring $R \subset R_{0}$ is called ideal-unimodal (IUS) if its maximal ideal admits generators $x, y$ whose (multi-) valuation satisfies the conditions of Table 1.

According to Theorem 2.1, ideal-unimodal (IUS) is the same as strictly unimodal for plane curve singularities of characteristic 0 . For char $\mathbf{k}>0$ we wish to reserve the name strictly unimodal (SUS) to singularities defined by their deformation properties (as in [1], [13]). As, at the time of this writing, the strictly unimodal singualrities have not been classified in positive characteristic, we have to distinguish between IUS and SUS for char $\mathbf{k}>0$.

We also use the following definition and notation.

Definition 1.6 Let $\left\{a_{1}, \ldots, a_{d}\right\}, a_{i} \in \mathfrak{m}$, represent a basis of $\mathfrak{m} /\left(\mathfrak{m} \cap t \mathfrak{m} R_{0}\right)$, $\mathbf{v}_{j}=\mathbf{v}\left(a_{j}\right)$. The set $\left\{\mathbf{v}_{1}, \ldots, \mathbf{v}_{d}\right\}$ will be called $a$ valuation type of $\mathrm{R}$ and denoted by $\operatorname{val}(\mathrm{R})$.

\section{Main theorem}

\subsection{Formulation}

We pass now to the main theorem. In addition to the notations 1.1 , let $\tilde{I}=$ $t^{2} \mathfrak{m} \mathrm{R}_{0}+\mathfrak{m}, \tilde{\mathrm{R}}=$ End $\tilde{I}$ and $\mathrm{A}_{0}$ the 4 -dimensional $\mathbf{k}$-algebra having a basis $\{1, a, b, a b\}$ with $a^{2}=b^{2}=0$ (these notations will be used only in the case char $\mathbf{k}=2$ ).

\footnotetext{
${ }^{1}$ In [1] these singularities are called "uni-" and "bimodal" (with respect to rightequivalence), while in [13] they are called "strictly unimodal" (with respect to contact equivalence). We use the latter terminology, which is more adequate in our situation.
} 
Table 1

\begin{tabular}{|c|c|c|c|c|c|}
\hline Type & $s$ & $\mathbf{v}(x)$ & $\mathbf{v}(y)$ & Condition & Name \\
\hline$E$ & $\begin{array}{l}1 \\
2\end{array}$ & $\begin{array}{c}(3) \\
(1,2) \\
(1,1,1)\end{array}$ & $\begin{array}{c}(l) \\
(\infty, l) \\
(\infty, k, l)\end{array}$ & $\begin{array}{l}l=7,8,10,11 \\
l=4,5,6,7 \\
l=2,3, k \geq l\end{array}$ & $\begin{array}{l}\mathrm{E}_{12}, \mathrm{E}_{14}, \mathrm{E}_{18}, \mathrm{E}_{20} \\
\mathrm{E}_{2,2 p-1}, \mathrm{E}_{13}, \mathrm{E}_{3,2 p-1} \\
(p \geq 1), \mathrm{E}_{19} \\
\mathrm{E}_{l, 2(k-l)}\end{array}$ \\
\hline $\mathrm{T}$ & $\begin{array}{l}2 \\
3 \\
4\end{array}$ & $\begin{array}{c}(2, k) \\
(1,1, k) \\
(1, \infty, 1, k)\end{array}$ & $\begin{array}{c}(l, 2) \\
(\infty, l, 2) \\
(\infty, 1, l, 1)\end{array}$ & $\begin{array}{l}k, l \text { odd, } l k>4 \\
k \text { odd, } l k \geq 4 \\
l k \geq 1\end{array}$ & $\begin{array}{l}\mathrm{T}_{k+2, l+2,2} \\
\mathrm{~T}_{k+2,2(l+1), 2} \\
\mathrm{~T}_{2(k+1), 2(l+1), 2}\end{array}$ \\
\hline W & $\begin{array}{l}2 \\
2\end{array}$ & $\begin{array}{c}(4) \\
(1,3) \\
(2,2) \\
(1,1,2)\end{array}$ & $\begin{array}{c}(l) \\
(\infty, l) \\
(3, l) \\
(\infty, 1,3)\end{array}$ & $\begin{array}{l}l=5,6,7 \quad(*) \\
l=4,5 \\
l=3 \\
l \geq 5, \text { odd } \\
l \geq 2\end{array} \quad(*)$ & $\begin{array}{l}\mathrm{W}_{12}, \mathrm{~W}_{1,2 p-1}^{\#} \\
(p \geq 1), \mathrm{W}_{18}^{\#} \\
\mathrm{~W}_{13}, \mathrm{~W}_{17} \\
\mathrm{~W}_{1,0}, \mathrm{~W}_{1,2 p}^{\#}(p \geq 1) \\
\mathrm{W}_{1, l-3} \\
\mathrm{~W}_{1,2 l-3}\end{array}$ \\
\hline Z & $\begin{array}{l}2 \\
3\end{array}$ & $\begin{array}{c}(1, l) \\
(1, \infty, 2) \\
(1, \infty, 1,1)\end{array}$ & $\begin{array}{c}(\infty, 3) \\
(\infty, 1, l) \\
(\infty, 1, l, k)\end{array}$ & $\begin{array}{l}l=4,5,7,8 \\
l=2,3,4,5 \\
l=1,2, k \geq l\end{array}$ & $\begin{array}{l}\mathrm{Z}_{11}, \mathrm{Z}_{13}, \mathrm{Z}_{17}, \mathrm{Z}_{19} \\
\mathrm{Z}_{0, p}, \mathrm{Z}_{12}, \mathrm{Z}_{1,2 p-1} \\
(p \geq 1), \mathrm{Z}_{18} \\
\mathrm{Z}_{l-1,2(k-l)}\end{array}$ \\
\hline
\end{tabular}

(*) If char $\mathbf{k}=2$, extra conditions in case $\mathrm{W}$ are required: if $s=1$ then $l=5$, if $s=2, v(x)=(2,2)$ and $l=3$ then $x^{2}-y^{3} \notin t^{7} \mathrm{R}_{0}$.

(This excludes $\mathrm{W}_{18}(s=1)$ and all $\mathrm{W}_{1, p}^{\#}(s=1$ or $s=2)$.) 
Theorem 2.1 Let $\mathrm{R}$ be a curve singularity. The following conditions are equivalent:

1. $\operatorname{par}(1, \mathrm{R}) \leq 1$.

2. $\mathrm{R}$ dominates a simple or ideal-unimodal plane curve singularity.

3. (a) $\mathrm{d}\left(\mathrm{R}_{0}\right) \leq 4$;

(b) $\mathrm{d}\left(\mathrm{R}_{1}\right) \leq 3$;

(c) $\mathrm{d}\left(\mathrm{R}_{2}+e \mathrm{R}\right) \leq 3$ for any idempotent $e \in \mathrm{R}_{0}$, such that $\mathrm{d}\left(e \mathrm{R}_{0}\right)=1$ (provided it exists);

(d) if $\mathrm{d}\left(\mathrm{R}_{0}\right)=3$, then $\mathrm{d}\left(\mathrm{R}_{3}\right) \leq 2$;

(e) if char $\mathbf{k}=2$, then $\tilde{R} / \tilde{I} \not \mathrm{A}_{0}$.

The proof of Theorem $2.1(3 . \Rightarrow 2$.) implies i) of the following corollary:

\section{Corollary 2.2}

i) Let $\mathrm{R}$ be a plane curve singularity. Then $\mathrm{R}$ is simple or ideal-unimodal if and only if the equivalent conditions of theorem 2.1 are satisfied.

ii) If char $\mathbf{k}=0$, then $\mathrm{R}$ dominates a simple or strictly unimodal plane curve singularity if and only if the equivalent conditions of Theorem 2.1 are satisfied.

Part ii) follows from the following parametric classification of strictly unimodal curve singularities.

Proposition 2.3 Let char $\mathbf{k}=0$ and $\mathrm{R}$ be a plane curve singularity. Then $\mathrm{R}$ is ideal-unimodal if and only if it is strictly unimodal.

Remark 2.4 One can see that the condition 3(c) of the theorem means that either $\mathrm{d}\left(\mathrm{R}_{2}\right) \leq 2$ or $e \mathfrak{m} \nsubseteq \mathfrak{m}+\mathfrak{m}^{3} R_{0}$.

It is perhaps worth giving a more geometric interpretation of these overring conditions.

- $\mathbf{R}_{0}=\prod_{i=1}^{s} \mathbf{k}\left[\left[t_{i}\right]\right]$ is the normalization of $\mathrm{R}$ and $\mathrm{d}_{0}=\mathrm{d}\left(\mathrm{R}_{0}\right)$ the usual multiplicity of the local ring $\mathrm{R}$.

- $\mathrm{R}_{1}=\mathbf{k}+\prod_{i=1}^{s} t_{2}^{m_{i}} \mathbf{k}\left[\left[t_{i}\right]\right]$ is the maximal local overring of $\mathrm{R}$, having the same multiplicity vector $\left(m_{1}, \ldots, m_{s}\right)$ as $\mathrm{R}$, where $m_{i}=$ multiplicity of the $i$-th branch; $\mathrm{d}_{1}=\mathrm{d}\left(\mathrm{R}_{1}\right)=\operatorname{dim}_{\mathbf{k}}\left(\mathrm{R}_{0} / \mathrm{R}_{1}\right)+1$.

- In general, we have $\mathrm{R}_{0} \supset \mathrm{R}_{1} \supset \mathrm{R}_{2} \supset \cdots \supset \mathrm{R}, \mathrm{R}_{i+1}=\mathbf{k}+\mathfrak{m} \mathrm{R}_{i}$, hence $\mathrm{d}_{i}=\mathrm{d}\left(\mathrm{R}_{i}\right)=\operatorname{dim}_{\mathbf{k}}\left(\mathrm{R}_{i+1} / \mathrm{R}_{i}\right)+1$. $\mathrm{R}_{i}$ is the maximal local overring of $\mathbf{R}$ such that $\mathrm{R}_{i} / \mathfrak{m}^{i} \mathrm{R}_{0}=\mathrm{R} / \mathfrak{m}^{i} \mathrm{R}_{0}$.

- If $e=\left(e_{1}, \ldots, e_{s}\right), e_{i} \in\{0,1\}$, is an idempotent such as $\mathrm{d}\left(e \mathrm{R}_{0}\right)=1$, then $e_{i}=1$ for some $i, e_{j}=0$ for $j \neq i$ and the $i$-th branch of $\mathrm{R}$ is nonsingular. Hence, $e \mathbf{R}=\mathbf{k}\left[\left[t_{i}\right]\right]$ and $\mathrm{d}\left(\mathrm{R}_{2}+e \mathrm{R}\right) \leq 3$ is a condition on the remaining branches of $\mathrm{R}$. 


\section{$2.2 \quad$ Proofs}

Proof of Theorem 2.1. 1. $\Longrightarrow$ 3. Suppose first that $d=\mathrm{d}_{0} \geq 5$. Consider the factoralgebra $A=\mathrm{R}_{0} / \mathfrak{m R}_{0}$. If $V$ is any subspace in $A$, then its pre-image $M(V)$ in $\mathrm{R}_{0}$ is an R-submodule. Moreover, if $V$ and $U$ are two subspaces in $A$ such that $A V=A U=A$, then, evidently, $M(V) \simeq M(U)$ if and only if $U=a V$ for some invertible element $a \in A$. Consider now the subset $\mathrm{Gr}_{0}(m, A)$ of the Grassmannian $\operatorname{Gr}(m, A)$, consisting of all such subspaces $V$ that $A V=A$. Obviously, it is an open subset, hence, an algebraic variety over $\mathbf{k}$ of dimension $m(d-m)$. The algebraic group $\mathrm{G}=A^{*} / k^{*}$ of dimension $d-1$ is acting on this variety, and different orbits of this action correspond to nonisomorphic R-ideals. In particular, there are families of non-isomorphic ideals of dimension $\geq \operatorname{dim} \mathrm{Gr}_{0}(m, A)-\operatorname{dim} \mathrm{G}=(m-1)(d-m-1)$ for $1 \leq m \leq d-1$. But, as $d>4, \operatorname{dim} \mathrm{G} \leq \operatorname{dim} \mathrm{Gr}_{0}(2, A)-2$. In view of [5] (Corollary 3.9), this implies $\operatorname{par}(1, R) \geq 2$.

Let now $d\left(R_{1}\right) \geq 4$. Note that $\operatorname{rad} R_{1}=m R_{0}$ and $R_{1} / \operatorname{rad} R_{1}=k$. Thus, the algebra $A^{\prime}=\mathrm{R}_{1} / \mathfrak{m} R_{1}$ is local with radical $\mathrm{J}=\mathfrak{m} \mathrm{R}_{0} / \mathfrak{m R}_{1}$. Moreover, $\mathfrak{m R}_{1} \supseteq\left(\mathfrak{m R}_{0}\right)^{2}$, whence $\mathrm{J}^{2}=0$. Then, for any subspace $W \subseteq \mathrm{J}$, the subspace $V=\mathbf{k}+W$ is a subalgebra in $A$ and its pre-image $M(V)$ is a subring of $\mathbf{R}_{0}$. Hence, taking different subspaces $W \subseteq \mathrm{J}$, we get non-isomorphic R-modules. As $\operatorname{dim} \mathrm{J}=\mathrm{d}_{1}-1, \operatorname{dim} \operatorname{Gr}(2, \mathrm{~J})=m\left(\mathrm{~d}_{1}-m-1\right)$ for $1 \leq m \leq \mathrm{d}_{1}$ and, hence, $\operatorname{par}(1, \mathrm{R}) \geq 2$, if $\mathrm{d}_{1} \geq 4$.

Remark 2.5 The same observations show that $\operatorname{par}(1, \mathrm{R}) \geq\left(\frac{\mathrm{d}_{0}-2}{2}\right)^{2}$ for $\mathrm{d}_{0}$ even respectively $\geq\left(\frac{d_{0}-1}{2}\right)\left(\frac{d_{0}-3}{2}\right)$ for $d_{0}$ odd and if $d_{0}=d_{1} \operatorname{par}(1, R) \geq \frac{d_{1}}{2}\left(\frac{d_{1}-2}{2}\right)$ for $d_{1}$ even respectively $\geq\left(\frac{d_{1}-1}{2}\right)^{2}$ for $d_{1}$ odd.

To complete the proof, we need two simple lemmas.

Lemma 2.6 Let $I, J \subset \mathrm{R}$ be full ideals. Then

$$
\operatorname{dim}(I / J I)=\operatorname{dim} \mathrm{R}_{0} / J \mathrm{R}_{0}-\operatorname{dim}(J I / r I)
$$

for any generator $r$ of the principal $\mathrm{R}_{0}$-ideal $J \mathrm{R}_{0}$.

Proof. Since $J$ is full, $r$ is a nonzero divisor of $\mathrm{R}_{0}$ and the snake lemma of

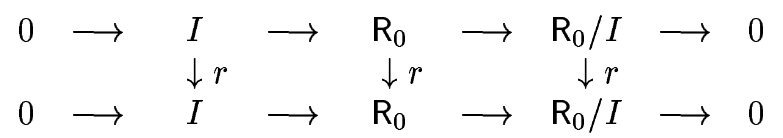

shows $\operatorname{dim} \mathrm{R}_{0} / r \mathrm{R}_{0}=\operatorname{dim} I / r I$ which is equal to $\operatorname{dim} I / J I+\operatorname{dim} J I / r I$.

Lemma 2.7 Suppose that $\mathrm{d}_{0}=\mathrm{d}_{1}=\cdots=\mathrm{d}_{k}, k \geq 1$. Let $r \in \mathfrak{m}$, such that $r \mathrm{R}_{0}=\mathrm{mR}_{0}$. Then 
1. $r, r^{2}, \ldots, r^{k}$ form a basis of $\mathfrak{m}_{k+1} / \mathfrak{m}^{k+1} \mathrm{R}_{0}$.

2. $r \mathfrak{m}_{i}=\mathfrak{m m}_{i}$ and $\operatorname{dim} \mathfrak{m}_{i} / \mathfrak{m m}_{i}=\mathrm{d}_{0}$ for $i=0, \ldots, k$.

Proof. To prove the first assertion, consider the dimensions

$$
c_{j}=\operatorname{dim}\left(\mathfrak{m}_{j} / \mathfrak{m}^{j} \mathrm{R}_{0}\right)
$$

and note that for $j>0$

$$
\mathrm{d}_{j}=1+\operatorname{dim}\left(\mathfrak{m}_{j} / \mathfrak{m}_{j+1}\right)=1+c_{j}+\operatorname{dim}\left(\mathfrak{m}^{j} \mathrm{R}_{0} / \mathfrak{m}^{j+1} \mathrm{R}_{0}\right)-c_{j+1} .
$$

Evidently, $\operatorname{dim}\left(\mathfrak{m}^{j} \mathrm{R}_{0} / \mathfrak{m}^{j+1} \mathrm{R}_{0}\right)=\mathrm{d}_{0}$ for all $j$. So, we have $c_{j+1}=c_{j}+1$ for $1 \leq j \leq k$, whence, $c_{j}=j-1,0 \leq j \leq k+1$. In particular, $\operatorname{dim}\left(\mathfrak{m}_{k+1} / \mathfrak{m}^{k+1} \mathrm{R}_{0}\right)=k$. As, of course, the elements $r, r^{2}, \ldots, r^{k}$ are linear independent modulo $\mathfrak{m}^{k+1} \mathrm{R}_{0}$, they form a basis of this vector space.

Now note that $r \mathfrak{m}_{k} \subseteq \mathfrak{m m}_{k}$ and $\operatorname{dim}\left(\mathfrak{m}_{k} / r \mathfrak{m}_{k}\right)=\operatorname{dim}\left(\mathrm{R}_{0} / \mathfrak{m R}_{0}\right)=\mathrm{d}_{0}$ in view of Lemma 2.6. But the result just obtained implies that

$$
\begin{aligned}
\operatorname{dim}\left(\mathfrak{m}_{k} / \mathfrak{m m}_{k}\right) & =\operatorname{dim}\left(\mathfrak{m}_{k} / \mathfrak{m}_{k+1}\right)+\operatorname{dim}\left(\mathfrak{m}_{k+1} / \mathfrak{m m}_{k}\right) \\
& =\mathrm{d}_{0}-1+\operatorname{dim}\left(\mathfrak{m}_{k+1} /\left(\mathfrak{m}^{2}+\mathfrak{m}^{k+1} \mathrm{R}_{0}\right)=\mathrm{d}_{0} .\right.
\end{aligned}
$$

Therefore, $r \mathfrak{m}_{k}=\mathfrak{m m}_{k}$

If $r^{j+1} b_{i} \in r^{j+1} \mathrm{R}_{0}, i=1, \ldots, l$, are a basis of $\mathfrak{m}_{j+1} / \mathfrak{m}_{j+2}=\left(\mathfrak{m}+r^{i+1} \mathrm{R}_{0}\right) /(\mathfrak{m}+$ $\left.r^{j+1} \mathrm{R}_{0}\right)$, then $r^{j} b_{i}$ are linear independent in $\mathfrak{m}_{j} / \mathfrak{m}_{j+1}$. Hence

Lemma $2.8 \mathrm{~d}_{j+1} \leq \mathrm{d}_{j}$ for every $j \geq 0$.

Now suppose that $\mathrm{d}_{0}=\mathrm{d}_{3}=3$. Consider the factoralgebra $F=\mathrm{R}_{2} / \mathfrak{m}^{2} \mathfrak{m}_{2}$. Choosing $r \in \mathfrak{m}$ as in Lemma 2.7, we see that $\mathfrak{m}^{i} \mathfrak{m}_{2}=r^{i} \mathfrak{m}_{2}$ for all $i$, $\operatorname{dim}\left(\mathfrak{m}_{2} / r \mathfrak{m}_{2}\right)=3$ and $\operatorname{dim} F=7$. Of course, $r \notin r \mathfrak{m}_{2}$, so we can choose $r, u, v \in \mathfrak{m}_{2}$ linear independent modulo $r \mathfrak{m}_{2}$. Then $\left\{1, r, u, v, r^{2}, r u, r v\right\}$ is a basis of $F$. Now $F$ contains a 2-parameter family of subalgebras, containing the image of $\mathrm{R}$ (i.e. 1 and $r$ ), namely, the subalgebras $A(\lambda, \mu)$ with bases $\left\{1, r, u+\lambda v+\mu r v, r^{2}, r u+\lambda r v\right\}$. Then their pre-images in $\mathrm{R}_{2}$ form a 2-parameter family of overrings of $\mathrm{R}$, hence, of pairwise non-isomorphic $\mathrm{R}$ ideals.

Remark 2.9 It follows from [3] that in this case we can only obtain families of overrings, as there are at most two non-isomorphic ideals with a fixed endomorphism ring.

At last, suppose that $\mathrm{d}\left(\mathrm{R}_{2}+e \mathrm{R}\right)=4$ for some idempotent $e \in \mathrm{R}_{0}$, such that $d\left(e R_{0}\right)=1$. As we have noted, this means: $d\left(R_{2}\right)=3$ and $e \mathfrak{m} \subseteq \mathfrak{m}+$ $\mathfrak{m}^{3} \mathrm{R}_{0}$. Of course, the idempotent $e$ is primitive and $e \mathrm{R}=e \mathrm{R}_{0}$. Denote $\mathrm{R}^{\prime}=$ $(1-e) \mathrm{R} ; \mathrm{R}_{k}^{\prime}=(1-e) \mathrm{R}_{k} ; \mathrm{d}_{k}^{\prime}=\mathrm{d}\left(\mathrm{R}_{k}^{\prime}\right)$. Then $\mathrm{d}_{0}^{\prime}=\mathrm{d}_{1}^{\prime}=\mathrm{d}_{2}^{\prime}=3$. Hence, 
we can apply Lemma 2.7 and choose an element $r \in \mathfrak{m}$, such that $\left\{r, r^{2}\right\}$ form a basis of $\mathfrak{m R}_{2}^{\prime} / \mathfrak{m}^{3} \mathrm{R}_{0}^{\prime}$. Consider the factoralgebra $F=\left(e \mathrm{R}+\mathrm{R}_{1}\right) /\left(\mathfrak{m}^{2}+\right.$ $\left.\mathfrak{m}^{3} \mathrm{R}_{0}\right)$. If $\{r, u, v\}$ is a basis of $\mathfrak{m R}_{0}^{\prime} / \mathfrak{m}^{2} \mathrm{R}_{0}^{\prime}$, then a basis of $F$ can be chosen in the form: $\{1, e, r, u, v, r u, r v\}$. The subspaces $V(\lambda)=V\left(\lambda_{0}, \lambda_{1}, \lambda_{2}\right)$ with the bases $\left\{1, e+\lambda_{0} u+\lambda_{1} v+\lambda_{2} r v, r, \lambda_{0} r u+\lambda_{1} r v\right\}$, where $\lambda_{0} \neq 0$, form a 3 dimensional family of R-submodules in $F$. Thus, they define a 3 -dimensional family $M(\lambda)$ of $R$-ideals. But it follows from [5] (proof of Proposition 3.6), that the ideals, isomorphic to some fixed $M(\lambda)$, form a subvariety of dimension $\operatorname{dim}(V(\lambda) / S(\lambda))$, where $S(\lambda)=\{a \in F \mid a V(\lambda) \subseteq V(\lambda)\}$. As $\operatorname{dim} V(\lambda)=4$ and $S(\lambda)\left\{1, r, \lambda_{0} r u+\lambda_{1} r v\right\}$, this dimension is at most (indeed, equals) 1 . Hence, again $\operatorname{par}(1, \mathrm{R}) \geq 2$.

If char $\mathbf{k}=2$ and $\tilde{\mathrm{R}} / \tilde{I} \simeq \mathrm{A}_{0}$, consider the subspaces $A(\lambda, \mu) \subset \mathrm{A}_{0}$ with bases $\{1, a+\lambda b+\mu a b\}$. They are subalgebras in $\mathrm{A}_{0}$ (as char $\mathbf{k}=2$ ), hence, their pre-images in $\tilde{R}$ form a 2-parameter family of overrings of $R$, hence, of non-isomorphic R-ideals

$3 \Longrightarrow 2$. Take any ring $\mathrm{R}$ satisfying the conditions $3(\mathrm{a}-\mathrm{e})$. It is known (cf. [3],[7])that if $\mathbf{R}$ has only finitely many non-isomorphic ideals then it dominates one of the simple pane curve singularities. So, we may supposethat $R$ has infinitely many non-isomorphic ideals, i.e. $\mathrm{d}_{0} \geq 3$ and if $\mathrm{d}_{0}=3$, then also $\mathrm{d}_{1}=3$ (cf. ibid.). Suppose first that $s=1$, where $s$ is the number of branches. Then the condition (a) implies that $\operatorname{val}(\mathrm{R})=\{3\}$ or $\{4\}$. In the first case the condition (d) easily implies that $R$ contains also an element of the valuation $l \in\{7,8,10,11\}$. But then it dominates a SUS of type $\mathrm{E}$ (cf. the list). If $\operatorname{val}(R)=\{4\}$, then the condition (b) impliesthat $R$ contains an element of the valuation $5 \leq l \leq 7$, hence, dominates a SUS of type $W$. If $\operatorname{char} \mathbf{k}=2$, the condition (e) implies also that $\mathrm{R}$ contains an element of the valuation 5 : otherwise $\tilde{I}=t^{6} \mathrm{R}_{0}+\mathbf{k}+\mathbf{k} t^{4}$, hence $\tilde{\mathrm{R}}=t^{2} \mathrm{R}_{0}+\mathbf{k}$ and $\tilde{\mathrm{R}} / \tilde{I} \simeq \mathrm{A}_{0}$.

Let now $s=2$. If $\mathrm{d}_{0}=3$ then $\operatorname{val}(\mathrm{R})=\{(1,2)\}$ (up to a numbering of the branches; later we omit this notice). Again the condition (d) implies that $\mathrm{R}$ contains an element of the valuation $(\infty, l)$ with $4 \leq l \leq 7$, hence, dominates a SUS of type $E$. Suppose that $d_{0}=4$. Then the following cases can occur:

- $\operatorname{val}(\mathrm{R}) \supset\{(1,3)\}$. Then the condition (b) implies that $\mathrm{R}$ contains an element with the valuation either $(\infty, 4)$ or $(\infty, 5)$, hence, dominate a SUS of type W.

- $\operatorname{val}(\mathrm{R}) \supset\{(2,2)\}$. Again (b) implies that $R$ contains an element with the valuation $(3, l)$, i.e. dominates a SUS of type $\mathrm{W}$. Again, if $\operatorname{char} \mathbf{k}=2$ and $x^{3}-y^{2} \in t^{7} \mathrm{R}_{0}$, we get that $\tilde{\mathrm{R}} / \tilde{I} \simeq \mathrm{A}_{0}$.

- $\operatorname{val}(\mathrm{R})=\{(2, k),(l, 2)\}$. Then $\mathrm{R}$ dominates a SUS of type $\mathrm{T}$.

- $\operatorname{val}(\mathrm{R})=\{(1, l),(\infty, 3)\}$. Now the condition (c) obviously implies that $l \leq 8$ (and not 6 ), hence, $\mathrm{R}$ dominates a SUS of type $\mathrm{Z}$. 
If $s=\mathrm{d}_{0}=3$, then $\operatorname{val}(\mathrm{R})=\{(1,1,1)\}$ and the condition (d) implies that $\mathrm{R}$ contains am element of the valuation $(\infty, 3, l)$, i.e. dominates a SUS of type $\mathrm{E}$. Let $s=3, \mathrm{~d}_{0}=4$. If $\mathrm{val}(\mathrm{R})$ consists of only one vector, then it is $(1,1,2)$ and the condition (b) implies immediately that $\mathrm{R}$ contains also an element of the valuation $(\infty, 3, l)$, hence, dominate a SUS of type $W$. If val (R) consists of 2 vectors, then there are the following possibilities:

- $\operatorname{val}(\mathrm{R})=\{(1,1, k),(\infty, l, 2)\}$. Then $\mathrm{R}$ dominates a SUS of type $\mathrm{T}$.

- $\operatorname{val}(\mathrm{R})=\{(1, \infty, l),(\infty, 1,2)\}$. Then the condition (c) implies that $l \leq 5$, hence, $\mathrm{R}$ dominates a SUS of type $\mathrm{Z}$.

If $\operatorname{val}(\mathrm{R})$ consists of 3 vectors, they may be chosen as

$$
\{(1, \infty, k),(\infty, 1, l),(\infty, \infty, 2)\}
$$

and $\mathrm{R}$ dominates a SUS of type $\mathrm{T}$.

At last, let $s=4$. The condition (b) implies that val (R) has at least 2 vectors. If there are really only 2 of them, then either $\operatorname{val}(\mathrm{R})=\{(1, \infty, k, 1),(\infty, 1,1, l)\}$ or $\operatorname{val}(\mathrm{R})=\{(1, \infty, 1,1),(\infty, 1, k, l)\}$. In the first case $\mathrm{R}$ dominates one of the SUS of type $\mathrm{T}$, while in the latter case the condition (c) implies that $k \leq 2$, thus $\mathrm{R}$ dominates a SUS of type $\mathrm{Z}$. Finally, if $\operatorname{val}(\mathrm{R})$ contains 3 vectors, one can easily see that $R$ dominates a SUS of type $T$

Proof of Proposition 2.3. The parametric form of an SUS, as computed by Schappert ([11]), satisfies the conditions of Table 1, hence it is an IUS.

Let $\mathrm{R}$ be an IUS. In the following subsection we classify curve singularities of multiplicity 3 and 4 given in parametric form. This implies the sixth column of Table 1 (notations of [1]), hence ideal-unimodal singularities are strictly unimodal.

\subsection{Classification in characteristic 0}

Let char $\mathbf{k}=0$. Any local ring of a plane curve singularity of multiplicity 3 or 4 admits generators $x, y$ with $v(x)$ and $v(y)$ as in columns 3 and 4 of Table 1, together with a few more cases. Starting from these valuations, we deduce a relation between $x$ and $y$ and determine its position in Arnold's list of singularities [1], classifying in this way all parametrizations of plane curve singularities of multiplicity 3 and 4 .

We proceed by increasing $s$, the number of branches.

One branch $(s=1)$ :

- (Multiplicity 3) $v(x)=3, v(y)=l$ :

after a change of the parametrization, we may assume (since char $\mathbf{k}=0$ ) that

$$
x=t^{3}, y=t^{l}+t^{m} p\left(t^{3}\right),
$$


$l \not \equiv 0(3), m+l \equiv 0(3), l \geq 4, p(0) \neq 0$. These satisfy the relation

$$
y^{3}-3 y x^{(l+m) / 3} p(x)-x^{l}-x^{m} p^{3}(x) .
$$

Following Arnold's determinator ([1], Para. 16), we obtain

$$
\begin{aligned}
& l=3 k+1 \Rightarrow \mathrm{E}_{6 k}, \\
& l=3 k+2 \Rightarrow \mathrm{E}_{6 k+2} .
\end{aligned}
$$

(Only $k=2,3$ give strictly unimodal singularities.)

- (Multiplicity 4) $v(x)=4, v(y)=l$ :

we may assume

$$
x=t^{4}, y=t^{4 k+1} p(t)+t^{4 n+2} q(t)+t^{4 m+3} r(t),
$$

$k, n, m \geq 1, p, q, r \in \mathbf{k}\left[\left[t^{4}\right]\right], l=\min \{4 k+1,4 n+2,4 m+3\}$.

We obtain the relation $(p, q, r \in \mathbf{k}[[x]])$,

$$
\begin{aligned}
& y^{4}-y^{2}\left(2 q^{2} x^{2 n+1}+4 p r x^{k+m+1}\right) \\
& \quad-y\left(4 p^{2} q x^{2 k+n+1}+4 q r^{2} x^{2 m+n+2}\right) \\
& \quad-p^{4} x^{4 k+1}+q^{4} x^{4 n+2}-4 p q^{2} r x^{k+2 n+m+2}+2 p^{2} r^{2} x^{2 k+2 m+2}-r^{4} x^{4 m+3} .
\end{aligned}
$$

The determinator gives

$$
\begin{aligned}
& l=4 k+1 \Rightarrow \mathrm{W}_{12 k}, \\
& l=4 n+2 \Rightarrow \mathrm{W}_{n, 2 i-1}^{\#}, \quad i=2(k-n) \text { if } k \leq m, \\
& l=2(m-n)+1 \text { if } k>m, \\
& l=4 m+3 \Rightarrow \mathrm{W}_{12 m+6} .
\end{aligned}
$$

(Only $k=n=m=1$ provide strictly unimodal singularities.)

Two branches $(s=2)$ :

- $($ Multiplicity 3) $v(x)=(1,2), v(y)=(\infty, l)$ :

$$
x=\left(t_{1}, t_{2}^{2}\right), y=\left(0, t_{2}^{l}+t_{2}^{m} p\left(t^{2}\right)\right),
$$

$l \geq 3, l+m \equiv 1(2), p(0) \neq 0$, with relation $(p \in K[[x]])$

$$
y\left(y^{2}+x^{2 k}-x^{2 k+2 j-1} p^{2}-2 x^{k} y\right) \text { if } l=2 k, m=2 k+2 j-1
$$

and

$$
y\left(y^{2}+x^{2 k+1}-x^{2(k+j)} p^{2}-2 x^{k+j} y p\right) \text { if } l=2 k+1, m=2(k+j) .
$$

$l=2 k, m=2 k+2 j-1 \Rightarrow \mathrm{E}_{k, 2 j-1}, j \geq 1\left(\mathrm{~J}_{k, 2 j-1}\right.$ in [1])

$l=2 k+1 \Rightarrow \mathrm{E}_{6 k+1}$.

(Strictly unimodal for $k=2,3, D_{4}$ for $l=2$.) 
- (Multiplicity 4, 2 singular branches, 2 tangents) $v(x)=(2, k), v(y)=$ $(l, 2)$ :

$$
x=\left(t_{1}^{2}, t_{2}^{2 m+1} p\left(t_{2}^{2}\right)+t_{2}^{2 m+2} q\left(t_{2}^{2}\right)\right), y=\left(t_{1}^{2 n+1}, t_{2}^{2}\right)
$$

$k=2 m+1, l=2 n+1, m \geq 1, n \geq 1$ with relation $(p, q \in \mathbf{k}[[y]], p(0) \neq$ $0, q(0) \neq 0)$

$$
\left(y^{2}-x^{2 n+1}\right)\left(x^{2}-y^{2 m+1} p^{2}+y^{m+2} y^{2}-2 x y^{m+1} q\right),
$$

\section{$\Rightarrow \mathrm{T}_{k+2, l+2,2}$}

(even for $k=1$ or $l=1$ if $k l>4$. Note that $\mathrm{T}_{3, p+6,2}=\mathrm{E}_{2, p}$ ).

- (Multiplicity 4, 1 singular branch, 1 tangent) $v(x)=(1,3), v(y)=$ $(\infty, l)$ :

$$
x=\left(t_{1}, t_{2}^{3}\right), y=\left(0, t_{2}^{3 k+1} p\left(t_{2}^{3}\right)+t_{2}^{3 n+2} q\left(t_{2}^{3}\right)+t_{2}^{3 m+3} r\left(t_{2}^{3}\right)\right)
$$

$k, n, m \geq 1, l=\min \{3 k+1,3 n+2,3 m+3\}$ with relation

$$
\begin{gathered}
y\left(y^{3}-x^{3 k+1} p^{3}-3 x^{k+n+1} y p q-x^{3 n+2} q^{3}-3 x^{m+1} y^{2} r\right. \\
\left.+3 x^{k+n+m+2} p q r+3 x^{2 m+2} y r^{2}-x^{3 m+3} r^{3}\right) .
\end{gathered}
$$

$l=3 k+1 \Rightarrow \mathrm{W}_{12 k+1}$,

$l=3 n+2 \Rightarrow \mathrm{W}_{12 n+5}$,

$l=3 m+3, n \geq k>m \Rightarrow \mathrm{Z}_{6(m+k)-5}^{m+1}$.

- (Multiplicity 4, 2 singular branches, 1 tangent) $v(x)=(2,2), v(y)=$ $(2 k+1, l)$ :

$$
x=\left(t_{1}^{2}, t_{2}^{2}\right), y=\left(t_{1}^{2 k+1}, t_{2}^{2 n+1} p\left(t_{2}^{2}\right)+t_{2}^{2 m} q\left(t_{2}^{2}\right)\right),
$$

$k \geq 1, l=\min \{2 n+1,2 m\}$ with relation $(p, q \in K[[x]], p(0) \neq 0, q(0) \neq 0)$

$$
\begin{aligned}
& \left(y^{2}-x^{2 k+1}\right)\left(y^{2}-x^{2 n+1} p^{2}-2 x^{m} y^{q}+x^{2 m} q^{2}\right) \text {. } \\
& k=n<m \quad \Rightarrow \quad \mathrm{W}_{k, 0}(p(0) \neq 1), \mathrm{W}_{k, 2 j}^{\#}(p(0)=1) \\
& \text { with } j=\operatorname{ord}\left(y^{2}\left(t_{2}\right)-t_{2}^{2 k+1}\right)-(2 k+1) \text {, } \\
& k<n, m \quad \Rightarrow \quad \mathrm{W}_{k, 2(n-k)} \text {, } \\
& k \geq m \geq 2 \quad \Rightarrow \quad Y_{r, s}^{m}(1 \leq s \leq r) \text {. }
\end{aligned}
$$

- (Multiplicity 4, 1 singular branch, 2 tangents) $v(x)=(1, l), v(y)=$ $(\infty, 3)$ :

$$
x=\left(t_{1}, t_{2}^{l}+t_{2}^{k} p\left(t_{2}^{3}\right)\right), y=\left(0, t_{2}^{3}\right),
$$

$k>l \geq 4, k+l \equiv 0(3)$, with relation $(p \in \mathbf{k}[[y]], p(0) \neq 0)$

$$
y\left(x^{3}-y^{l}-y^{k} p^{3}-3 x y^{(k+l) / 3} p\right) .
$$

$l=3 p+1 \Rightarrow \mathrm{Z}_{6 p+5}$,

$l=3 p+2 \Rightarrow \mathrm{Z}_{6 p+7}$

$\left(l=3: \mathrm{E}_{6 j}(\right.$ if $k=3 j+1), \mathrm{E}_{6 j+2}($ if $\left.k=3 j+2)\right)$. 
Three branches $(s=3)$ :

- (Multiplicity 3, 1 tangent) $v(x)=(1,1,1), v(y)=(\infty, l, k)$ :

$$
x=\left(t_{1}, t_{2}, t_{3}\right), y=\left(0, t_{2}^{l}, t_{3}^{l+n} p\left(t_{3}\right)\right),
$$

$l \geq 2, n \geq 0$, with relation $(p \in K[[x]], p(0) \neq 0)$

$$
y\left(x^{l}-y\right)\left(x^{l+n} p-y\right) .
$$

$n=0 \Rightarrow \mathrm{E}_{l, 0}(p(0) \neq 1)$,

$n>0 \Rightarrow \mathrm{E}_{l, 2 n}\left(\mathrm{~J}_{l, 2 n}\right.$ in [1].)

- (Multiplicity 4, 2 smooth branches with same tangent, 2 tangents) $v(x)=$ $(1,1, k), v(y)=(\infty, l, 2)$ :

$$
x=\left(t_{1}, t_{2}, t_{3}^{k} q\left(t_{3}^{2}\right)\right), y=\left(0, t_{2}^{l} p\left(t_{2}\right), t_{3}^{2}\right),
$$

$k$ odd, $k l \geq 2 ;$ relation $(p(0) \neq 0, q(0) \neq 0)$

$$
y\left(y-x^{l} p(x)\right)\left(x^{2}-y^{k} q^{2}(y)\right) .
$$

$\Rightarrow \mathrm{T}_{k+2,2(l+1), 2}$.

- (Multiplicity 4, 2 smooth branches, 1 tangent) $v(x)=(1,1,2), v(y)=$ $(\infty, l, 2 n+1)$,

$$
x=\left(t_{1}, t_{2}, t_{3}^{2}\right), y=\left(0, t_{2}^{l} p\left(t_{2}\right), t_{3}^{2 n+1} q\left(t_{3}^{2}\right)\right),
$$

$l \geq 2, n \geq 1 ;$ relation $(p(0) \neq 0, q(0) \neq 0)$

$$
y\left(y-x^{l} p(x)\right)\left(y^{2}-x^{2 n+1} q^{2}(x)\right),
$$

$l \geq n+1 \Rightarrow \mathrm{W}_{n, 2(l-n)-1}$,

$l \leq n \Rightarrow \mathrm{Z}_{6(n-l)}^{12 l}$.

- (Multiplicity 4, 2 smooth branches with different tangents, 2 tangents) $v(x)=(1, \infty, 2), v(y)=(\infty, 1,2 n+1)$ :

$$
x=\left(t_{1}, 0, t_{3}^{2}\right), y=\left(0, t_{2}, t_{3}^{l}+t_{3}^{k} q\left(t_{3}^{2}\right)\right),
$$

$k>l \geq 2$, relation $(q(0) \neq 0)$ for $l=2 j+1, k$ even:

$$
x y\left(y^{2}-x^{l}-x^{k} q^{2}-2 y x^{k / 2} q+2 x^{k} q^{2}\right) .
$$

$\Rightarrow \mathrm{Z}_{6 j+6}$,

for $l=2 j$ we have the relation

$$
\begin{gathered}
x y\left(y^{2}+x^{l}-x^{k} q^{2}-2 x^{j} y\right) \\
\Rightarrow \mathrm{Z}_{j-1, k-l}\left(\mathrm{Z}_{0, p}=X_{1, p}=\mathrm{T}_{4,4+p, 2}\right) .
\end{gathered}
$$


Four branches $(s=4)$ :

- (4 tangents, 3 tangents, or 2 tangents, each belonging to 2 branches) $v(x)=(1, \infty, 1, k), v(y)=(\infty, 1, l, 1)$ :

$$
x=\left(t_{1}, 0, t_{3}, t_{4}^{k} p\left(t_{4}\right)\right) y=\left(0, t_{2}, t_{3}^{l}, t_{4}\right),
$$

$k, l \geq 1 ;$ relation

$$
y x\left(x^{l}-y\right)\left(x-y^{k} p\right) .
$$

$\Rightarrow \mathrm{T}_{2(k+1), 2(l+1), 2}$.

- $(2$ tangents, one belonging to 3 branches $) \quad v(x)=(1, \infty, 1,1), v(y)=$ $(\infty, 1, l, k)$ :

$$
x=(t, 0, t, t), y=\left(0, t, t^{l} q(t), t^{k} p(t)\right),
$$

$k \geq l \geq 1 ;$ relation $(p(0) \neq 0, q(0) \neq 0)$

$$
y x\left(x^{l} q-y\right)\left(x^{k} p-y\right) .
$$

$\Rightarrow \mathrm{Z}_{l-1,2(k-l)}$.

- $(1$ tangent $) v(x)=(1,1,1,1), v(y)=(\infty, k, l, m)$ :

$$
x=\left(t_{1}, t_{2}, t_{3}, t_{4}\right), y=\left(0, t^{k}, t^{l} p(t), t^{m} q(t)\right),
$$

$1<k \leq l \leq m$; relation $(p(0) \neq 0, q(0) \neq 0)$

$$
y\left(x^{k}-y\right)\left(x^{l} p-y\right)\left(x^{m} q-y\right) .
$$

$k<l \leq m \quad \Rightarrow \quad \mathrm{Z}_{l-k-1,2(m-l)}^{k}$,

$k=l<m \quad \Rightarrow \quad \mathrm{X}_{k, j}(j \geq 0)$ or $\mathrm{Y}_{r, s}^{k}(1 \leq s \leq r)$.

(None is strictly unimodal.)

\section{Ideals of ideal-unimodal plane curve singular- ities}

Now we have to prove the implication $2 \Longrightarrow 1$, that is to show that any IUS from Table 1 has only 1-parameter families of ideals. Indeed, this was done in Schappert's paper [12]. Though Schappert supposed that char $\mathbf{k}=0$ and used another definition of IUS, one can check that his calculations are valid for our list too, independent of the characteristics. To demonstrate it and because Schappert's thesis has not been published, we show below examples of such calculations (in somewhat different form). Moreover, we have chosen the most complicated cases. 
Our calculations are based on the following simple observation (cf. [3]). Let $\mathrm{R}$ be a curve singularity, $\mathfrak{m}$ its maximal ideal and $\mathrm{S}=\operatorname{End}_{\mathrm{R}}(\mathfrak{m})=$ $\{a \in \mathrm{Q} \mid a \mathfrak{m} \subseteq \mathfrak{m}\}$. We keep these notations through the whole section and also put $\mathfrak{n}=\operatorname{rad} S, \mathrm{~S}^{\prime}=\operatorname{End}_{\mathbf{S}}(\mathfrak{n})$. For any R-ideal $I, \mathrm{~S} I$ is an S-ideal and $\mathfrak{m} I=\mathfrak{m S} I \subset I \subseteq \mathrm{S} I$. Consider the vector space $V=V(I)=I / \mathfrak{m} I$. It is a $g e n$ erating subspace in $W=\mathrm{S} I / \mathfrak{m S} I$, i.e. such that $F V=W$, where $F=\mathrm{S} / \mathfrak{m}$, and one can easily check that $I \simeq I^{\prime}$ if and only if $\bar{\gamma} V(I)=V\left(I^{\prime}\right)$ for some map $\bar{\gamma}: F \rightarrow F$ induced by an automorphisms $\gamma$ of $\mathrm{S} I$. Moreover, $\mathrm{S} \neq \mathrm{R}$, whenever $\mathrm{R}$ is not a discrete valuation ring. Therefore, we can calculate the ideals "inductively", ascending by overrings. Note also that any plane curve singularity $R$ is Gorenstein [2], i.e. inj. $\operatorname{dim}_{R} R=1$. Hence, $R$ has as only minimal overring $\mathrm{R}^{\prime}$ and any $\mathrm{R}$-ideal is either principal or an $\mathrm{R}^{\prime}$-ideal. In particular, $\operatorname{par}(1, \mathrm{R})=\operatorname{par}\left(1, \mathrm{R}^{\prime}\right)$.

Note that all IUS of type $\mathrm{T}$ are known to be tame, i.e. have at most 1parameter families of indecomposable torsion-free modules of any rank [4]. So we have to consider only the IUS of types W, Z and $\mathrm{E}$.

\subsection{Ideals of singularities of type $\mathrm{W}$}

Here we consider the case, when $s=1$ and $\mathrm{R}$ contains elements $x, y$ with $v(x)=4, v(y)=7$ ("type $\mathrm{W}_{18}$ " in Arnold's classification). It is convenient to suppose here that $t=x^{2} / y$. Of course, we suppose also that char $\mathbf{k} \neq 2$. It is easy to verify that then $\mathrm{R} \supset t^{18} \mathrm{R}_{0}$ and, if $\mathrm{R}$ is a plane curve singularity, its minimal overring contains even $t^{14} \mathrm{R}_{0}$. Hence, we may restrict ourselves by the case, when $\mathrm{R}$ is the smallest subalgebra of $\mathrm{R}_{0}$ containing $t^{14} \mathrm{R}_{0}$ and generated modulo $t^{14} \mathrm{R}_{0}$ by $x$ and $y$. Thus,

$$
\mathrm{R}=\left\langle 1, x, y, x^{2}, x y, x^{3}\right\rangle+t^{14} \mathrm{R}_{0},
$$

where, as usual, we denote by $\left\langle a_{1}, \ldots, a_{m}\right\rangle$ the $k$-subspace generated by $a_{1}, \ldots, a_{m}$. An obvious calculation shows that in this case

$$
\mathrm{S}=\left\langle 1, x, y, x^{2}\right\rangle+t^{10} \mathrm{R}_{0}
$$

and

$$
\mathrm{S}^{\prime}=\langle 1, z, x\rangle+t^{6} \mathrm{R}_{0},
$$

where $z=y / x$. As $v(z)=3$, it follows from [6] or [9] that $\mathrm{S}^{\prime}$ has only finitely many non-isomorphic ideals (it is the simple plane curve singularity of type $\left.E_{6}\right)$. Moreover, in these articles the precise list of such ideals is given. Namely, they are, except $R_{0}$ and $S^{\prime}$ itself:

$$
A=S^{\prime}\left\langle 1, t^{5}\right\rangle, \quad A^{*}=S^{\prime}\langle 1, t\rangle, \quad A^{\prime}=S^{\prime}\left\langle 1, t^{2}\right\rangle .
$$

Here $A$ and $A^{\prime}$ are overrings of $S^{\prime}$ and $A^{*}$ the module dual to $A$ with respect to the duality described e.g. in [3]. 
Now, for each of these ideals, say $M$, we have to calculate $M / \mathfrak{n} M$. Here is their list (we write " $r$ " for the image of an element $r \in M$ in $M / \mathfrak{n} M$ too):

$$
\begin{gathered}
\mathrm{S}^{\prime}=\left\langle 1, z, z^{2}, z^{3}\right\rangle, \mathrm{A}=\left\langle 1, z, t^{5}, t^{6}\right\rangle, \mathrm{A}^{*}=\left\langle 1, t, z, t^{6}\right\rangle, \\
\mathrm{A}^{\prime}=\left\langle 1, t^{2}, z, t^{5}\right\rangle, \mathrm{R}_{0}=:\left\langle 1, t, t^{2}, t^{3}\right\rangle .
\end{gathered}
$$

Now one can easily write down all generating subspaces $V$ from each $W=$ $M / \mathfrak{n} M$ (up to automorphisms of $M$ ). Consider the case $M=\mathrm{A}^{*}$ (the most complicated). First, as $V$ is generating, it has to contain at least two elements of the form: $a_{1}=1+\lambda_{1} z+\lambda_{2} t^{5}$ and $a_{2}=t^{2}+\mu_{1} z+\mu_{2} t^{5}$. Dividing by $a_{1}$ (which is the image of an invertible element from $A^{\prime}$ ), we may suppose that $a_{1}=1$. Suppose that $V=\left\langle a_{1}, a_{2}\right\rangle$. If $\mu_{1} \neq 0$, one can replace $V$ by $\left(1-\left(\mu_{1}+\mu_{2}\right)\left(2 \mu_{1}\right)^{-1}\right) V$, thus obtaining a subspace of the same form but with $\mu_{2}=0$. Hence, we get two 1-parameter families of S-ideals:

$$
F_{1}(\mu)=\mathrm{S}\left\langle 1, t^{2}+\mu z\right\rangle, \quad F_{2}(\mu)=\mathrm{S}\left\langle 1, t^{2}+\mu t^{5}\right\rangle .
$$

Suppose now that $V$ contains another element, which can be chosen in the form $b=\eta_{1} z+\eta^{2} t^{5}$. If $\eta_{1} \neq 0$, we may suppose that $\mu_{1}=0$ and then, multiplying by $1-\eta_{2} / \eta_{1} z$, also $\eta_{2}=0$, i.e. $b=z$. But then, multiplying by $1-\mu_{2} t^{3}$, we get $\mu_{2}=0$, which gives only one more ideal:

$$
I_{1}=\mathrm{S}\left\langle 1, t^{2}, z\right\rangle .
$$

If $\eta_{1}=0$, we may suppose $\mu_{2}=0$, obtaining 1-parameter family:

$$
F_{3}(\mu)=\mathrm{S}\left\langle 1, t^{2}+\mu z, t^{5}\right\rangle .
$$

Of course, we have to add to those families also the ideal $A^{\prime}$ itself (corresponding to $V=W)$. But we can remark that $\mathrm{d}\left(\mathrm{A}^{\prime}\right) \leq 4$, hence $\operatorname{dim} \mathrm{A}^{\prime} / \mathfrak{m A}^{\prime} \leq 4$ and $\mathfrak{m} A^{\prime}=\mathfrak{n} A^{\prime}$. Thus, any generating subspace in $A^{\prime} / \mathfrak{m} A^{\prime}$ (with respect to S) must coincide with $A^{\prime} / \mathfrak{m} A^{\prime}$ itself. Therefore, we need not consider the case $\mathrm{S} I=\mathrm{A}^{\prime}$ when calculating the R-ideals. The same argument is valid, of course, in each case, when we have an S-ideal $L$ such that $\mathfrak{n} L=\mathfrak{m} L$ (it is always the case, if $\left.\operatorname{dim}(L / \mathfrak{n S})=\mathrm{d}_{0}\right)$ : we may exclude them while calculating the R-ideals. In particular, here we may exclude all $\mathrm{S}^{\prime}$-ideals.

Quite analogous observations give us the following list of S-ideals (which are not $S^{\prime}$-ideals):

$\mathrm{S}^{\prime} L=\mathrm{S}^{\prime}$ :

$$
\begin{aligned}
& F_{4}(\mu)=\mathrm{S}\left\langle 1, z+\mu z^{3}\right\rangle, \quad F_{5}(\mu)=\mathrm{S}\left\langle 1, z^{2}+\mu z^{3}\right\rangle, \quad \mathrm{S}=\mathrm{S}\langle 1\rangle, \\
& I_{2}=\mathrm{S}\left\langle 1, z^{3}\right\rangle, \quad I_{3}=\mathrm{S}\left\langle 1, z, z^{2}\right\rangle, \quad I_{4}=\mathrm{S}\left\langle 1, z, z^{3}\right\rangle, \quad I_{5}=\mathrm{S}\left\langle 1, z^{2}, z^{3}\right\rangle .
\end{aligned}
$$

$\mathrm{S}^{\prime} L=\mathrm{A}$ :

$$
\begin{aligned}
& F_{6}(\mu)=\mathrm{S}\left\langle 1, z+\mu t^{5}\right\rangle, \mu \neq 0, \quad F_{7}(\mu)=\mathrm{S}\left\langle 1, z+\mu t^{5}, t^{6}\right\rangle, \mu \neq 0, \\
& F_{8}(\mu)=\mathrm{S}\left\langle 1, t^{5}+\mu t^{6}\right\rangle, \quad F_{9}(\mu)=\mathrm{S}\left\langle 1, z, t^{5}+\mu t^{6}\right\rangle, \quad I_{6}=\mathrm{S}\left\langle 1, t^{5}, t^{6}\right\rangle .
\end{aligned}
$$


$\mathrm{S}^{\prime} L=\mathrm{A}^{*}$ :

$$
F_{10}(\mu)=\mathrm{S}\langle 1, t+\mu z\rangle, \quad F_{11}(\mu)=\mathrm{S}\left\langle 1, t+\mu z, t^{6}\right\rangle, \quad I_{7}=\mathrm{S}\langle 1, t, z\rangle .
$$

$\mathrm{S}^{\prime} L=\mathrm{R}_{0}$ :

$$
I_{8}=\mathrm{S}\left\langle 1, t, t^{2}\right\rangle \text {. }
$$

Now we can pass to R-ideals $I$. Put $M=\mathrm{S} I$. It would be one of the ideals $F_{1-11}(\mu), I_{1-8}$ or $\mathrm{S}$. One can easily check that in the following cases $\mathfrak{m} M=$ $\mathfrak{n} M$, so we need not to consider them:

$$
\begin{aligned}
F_{i}(\mu) & \text { for } i \neq 4,8,10, \\
F_{i}(\mu) & \text { for } i=4,10 \text { and } \mu \neq 0 \\
I_{i} & \text { for } i \neq 2 .
\end{aligned}
$$

In the case $M=F_{4}(0)$ we have: $M / \mathfrak{m} M=\left\langle 1, z, t^{13}\right\rangle$. Hence, the only proper generating subspace is $\langle 1, z\rangle$, which gives one new ideal:

$$
I_{9}=\mathrm{R}\langle 1, z\rangle .
$$

Analogously, in the case $M=F_{10}(0)$ we obtain also one new ideal:

$$
I_{10}=\mathrm{R}\langle 1, t\rangle .
$$

In the case $M=F_{8}$ we have $M / \mathfrak{m} M=\left\langle 1, t^{5}+\mu t^{6}, t^{10}\right\rangle$. Hence, there is again only one proper generating subspace, namely, $\left\langle 1, t^{5}+\mu t^{6}\right\rangle$ and we obtain a new 1-parameter family:

$$
F_{12}(\mu)=\mathrm{R}\langle 1, u(\mu)\rangle, \quad \text { where } u(\mu)=t^{5}+\mu t^{6} .
$$

In the case $M=\mathrm{S}$ we have: $M / \mathfrak{m} M=\left\langle 1, t^{10}, t^{13}\right\rangle$. As there is no element with valuation 3 in $S$, we get here a new family parametrized by the projective line:

$$
F_{13}\left(\lambda_{0}: \lambda_{1}\right)=\mathrm{R}\left\langle 1, \lambda_{0} t^{10}+\lambda_{1} t^{13}\right\rangle
$$

At last, the case $M=I_{2}$ also gives a new 1-parameter family:

$$
F_{14}(\mu)=\mathrm{R}\left\langle 1, z^{3}+\mu t^{10}, t^{13}\right\rangle .
$$

Thus, we have described all R-ideals and proved that $\operatorname{par}(1, R)=1$. Quite similar (mainly easier) calculations show that $\operatorname{par}(1, \mathrm{R})=1$ all other singularities of type $\mathrm{W}$.

Remark. In the list of Schappert [11] the ideals $F_{2}(\mu)$ (which are indeed overrings of R) and $I_{3}$ are missing. 


\subsection{Ideals of singularities of type $Z$}

Now consider the singularities of type $\mathrm{Z}$. Here we suppose that $s=2$ and $\mathrm{R}$ contains elements $x, y$ with $\mathbf{v}(x)=(1,8), \mathbf{v}(y)=(\infty, 3)$ (IUS of type $\mathrm{Z}_{19}$ ). One can check that in this case $\mathrm{R} \supset\left(t_{1}^{3}, t_{2}^{17}\right) \mathrm{R}_{0}$ and, moreover, its minimal overring contains $\left(t_{1}^{2}, t_{2}^{14}\right)$. Hence, we may suppose that

$$
\mathrm{R}=\left\langle 1, x, y, y^{2}, y^{3}, x y, y^{4}\right\rangle+\left(t_{1}^{2}, t_{2}^{14}\right) \mathrm{R}_{0} .
$$

It is convenient to take for $t_{1}$ the first component of $x$ and choose $t_{2}$ in such way that $y^{3}=\left(0, t_{2}\right) x$. Now

$$
\mathrm{S}=\left\langle 1, x, y, y^{2}, y^{3}\right\rangle+\left(t_{1}, t_{2}^{11}\right) \mathrm{R}_{0} \quad \text { and } \quad \mathrm{S}^{\prime}=\mathrm{D}_{1} \oplus \mathrm{S}_{2},
$$

where $\mathrm{D}_{1}=\mathbf{k}\left[\left[t_{1}\right]\right]$ and $\mathrm{S}_{2}=\mathbf{k}\left[\left[t_{2}^{3}, t_{2}^{5}\right]\right]$ (the simple plane curve singularity of type $\mathrm{E}_{8}$ ). Here is the list of all $\mathrm{S}_{2}$-ideals (cf. [6],[9]) given by their generators over $\mathrm{S}_{2}$ and over $\mathrm{S}$ :

\begin{tabular}{|c|c|c|}
\hline Ideal & $\mathrm{S}_{2}$-generators & S-generators \\
\hline & & \\
$\mathrm{S}_{2}$ & 1 & $1, t_{2}^{5}, t_{2}^{10}$ \\
$\mathrm{~A}$ & $1, t_{2}^{7}$ & $1, t_{2}^{5}, t_{2}^{7}$ \\
$\mathrm{~A}^{*}$ & $1, t_{2}^{2}$ & $1, t_{2}^{2}, t_{2}^{7}$ \\
$\mathrm{~B}$ & $1, t_{2}^{4}$ & $1, t_{2}^{4}, t_{2}^{5}$ \\
$\mathrm{~B}^{*}$ & $1, t_{2}$ & $1, t_{2}, t_{2}^{5}$ \\
$\mathrm{~B}^{\prime}$ & $1, t_{2}^{2}, t_{2}^{4}$ & $1, t_{2}^{2}, t_{2}^{4}$ \\
$\mathrm{D}_{2}$ & $1, t_{2}, t_{2}^{2}$ & $1, t_{2}, t_{2}^{2}$ \\
& & \\
\hline
\end{tabular}

Any (full) $\mathrm{S}^{\prime}$-ideal is of the form $\mathrm{D}_{1} \oplus N$, where $N$ is an $\mathrm{S}_{2}$-ideal. Consider first the S-ideals $I$ such that $\mathrm{S}^{\prime} I=\mathrm{D}_{1} \oplus \mathrm{A}$. Then $\mathfrak{n} I=\mathfrak{n}+\left\langle\left(0, t_{2}^{10}\right)\right\rangle$,

$$
\mathrm{S}^{\prime} I / \mathfrak{n} I=W=\left\langle(1,0),(0,1),\left(0, t_{2}^{5}\right),\left(0, t_{2}^{7}\right)\right\rangle
$$

and any generating subspace $V \subseteq W$ contains an element of the form $(1, a)$ and also elements of the form $\left(\alpha_{1}, 1+\mu_{1} t_{2}^{5}\right),\left(\alpha_{2}, t_{2}^{7}+\mu_{2} t_{2}^{5}\right)$. Multiplying by $\left(1,1-\mu_{1} t_{2}^{5}\right)$, we may suppose that $V$ contains $\left(\alpha_{1}, 1\right)$. Then, if $\operatorname{dim} V=2$, there are only two possibilities:

$$
V=\left\langle(1,1),\left(0, \mu t_{2}^{5}+t_{2}^{7}\right)\right\rangle \text { and } V=\left\langle(0,1),\left(1, \mu t_{2}^{5}+t_{2}^{7}\right)\right\rangle .
$$

If $\operatorname{dim} V=3$, we can add also an element of the form $\left(\beta, t_{2}^{5}\right)$ or $(1,0)$ It gives three more possibilities:

$$
\begin{gathered}
V=\left\langle(1,1),\left(0, t_{2}^{5}\right),\left(0, t_{2}^{7}\right)\right\rangle, \quad V=\left\langle(0,1),\left(\lambda_{0}, t_{2}^{5}\right),\left(\lambda_{1}, t_{2}^{7}\right)\right\rangle \\
V=\left\langle(1,0),(0,1),\left(\mu t_{2}^{5}+t_{2}^{7}\right)\right\rangle
\end{gathered}
$$


where $\left(\lambda_{0}: \lambda_{1}\right)$ is a point of the projective line. Hence, here is the list of the corresponding S-ideals (which are not $\mathrm{S}^{\prime}$-ideals):

$$
\begin{aligned}
F_{1}(\mu) & =\mathrm{S}\left\langle(1,1),\left(0, \mu t_{2}^{5}+t_{2}^{7}\right)\right\rangle, \\
F_{2}(\mu) & =\mathrm{S}\left\langle(0,1),\left(1, \mu t_{2}^{5}+t_{2}^{7}\right)\right\rangle, \\
F_{3}\left(\lambda_{0}: \lambda_{1}\right) & =\mathrm{S}\left\langle(0,1),\left(\lambda_{0}, t_{2}^{5}\right),\left(\lambda_{1}, t_{2}^{7}\right)\right\rangle, \\
F_{4}(\mu) & =\mathrm{S}\left\langle(1,0),(0,1),\left(\mu t_{2}^{5}+t_{2}^{7}\right)\right\rangle, \\
I_{1} & =\mathrm{S}\left\langle(1,1),\left(0, t_{2}^{5}\right),\left(0, t_{2}^{7}\right)\right\rangle .
\end{aligned}
$$

Note that the ideals $F_{4}(\mu)$ are decomposable (as modules), while all other ideals of this list are indecomposable.

Analogous calculations give the following list of all S-ideals $I$, which are not $\mathrm{S}^{\prime}$-ideals:

$$
\mathrm{S}^{\prime} I=\mathrm{S}^{\prime}:
$$

$$
\begin{aligned}
F_{5}\left(\lambda_{0}: \lambda_{1}\right) & =\mathrm{S}\left\langle\left(\lambda_{0}, 1\right),\left(\lambda_{1}, t_{2}^{5}\right)\right\rangle, \\
\mathrm{S} & =\mathrm{S}\langle(1,1)\rangle, \\
I_{2} & =\mathrm{S}\left\langle(1,1),\left(0, t_{2}^{10}\right)\right\rangle, \\
I_{3} & =\mathrm{S}\left\langle(1,1),\left(0, t_{2}^{5}\right),\left(0, t_{2}^{10}\right)\right\rangle, \\
I_{4} & =\mathrm{S}\left\langle(0,1),\left(1, t_{2}^{5}\right),\left(0, t_{2}^{10}\right)\right\rangle, \\
I_{5} & =\mathrm{S}\left\langle(0,1),\left(1, t_{2}^{10}\right)\right\rangle, \\
I_{6} & =\mathrm{S}\left\langle(0,1),\left(0, t_{2}^{5}\right),\left(1, t_{2}^{10}\right)\right\rangle, \\
I_{7} & =\mathrm{S}\langle(1,0),(0,1)\rangle, \\
I_{8} & =\mathrm{S}\left\langle(1,0),(0,1),\left(0, t_{2}^{5}\right)\right\rangle, \\
I_{9} & =\mathrm{S}\left\langle(1,0),(0,1),\left(0, t_{2}^{10}\right)\right\rangle .
\end{aligned}
$$

$\mathrm{S}^{\prime} I=\mathrm{D}_{1} \oplus \mathrm{A}^{*}, \mathfrak{n} I=\mathfrak{n}+\left\langle\left(0, t_{2}^{5}\right),\left(0, t_{2}^{10}\right)\right\rangle:$

$$
\begin{aligned}
F_{6}\left(\lambda_{0}: \lambda_{1}\right) & =\mathrm{S}\left\langle\left(\lambda_{0}, 1\right),\left(\lambda_{1}, t_{2}^{2}\right)\right\rangle, \\
F_{7}\left(\lambda_{0}: \lambda_{1}\right) & =\mathrm{S}\left\langle\left(\lambda_{0}, 1\right),\left(\lambda_{1}, t_{2}^{2}\right),\left(0, t_{2}^{7}\right)\right\rangle, \\
I_{10} & =\mathrm{S}\left\langle(0,1),\left(0, t_{2}^{2}\right),\left(1, t_{2}^{7}\right)\right\rangle, \\
I_{11} & =\mathrm{S}\left\langle(1,0),(0,1),\left(0, t_{2}^{2}\right)\right\rangle .
\end{aligned}
$$

$\mathrm{S}^{\prime} I=\mathrm{D}_{1} \oplus \mathrm{B}, \mathfrak{n} I=\mathfrak{n}+\left\langle\left(0, t_{2}^{7}\right),\left(0, t_{2}^{10}\right)\right\rangle:$

$$
\begin{aligned}
F_{8}(\mu) & =\mathrm{S}\left\langle(1,1),\left(0, t_{2}^{4}+\mu t_{2}^{5}\right)\right\rangle, \\
F_{9}(\mu) & =\mathrm{S}\left\langle(0,1),\left(1, t_{2}^{4}+\mu t_{2}^{5}\right)\right\rangle, \\
F_{10}\left(\lambda_{0}: \lambda_{1}\right) & =\mathrm{S}\left\langle(0,1),\left(\lambda_{0}, t_{2}^{4}\right),\left(\lambda_{1}, t_{2}^{5}\right)\right\rangle, \\
F_{11}(\mu) & =\mathrm{S}\left\langle(1,0),(0,1),\left(0, t_{2}^{4}+\mu t_{2}^{5}\right)\right\rangle, \\
I_{12} & =\mathrm{S}\left\langle(1,1),\left(0, t_{2}^{4}\right),\left(0, t_{2}^{5}\right)\right\rangle .
\end{aligned}
$$




$$
\begin{aligned}
\mathrm{S}^{\prime} I=\mathrm{D}_{1} \oplus \mathrm{B}^{*}, \mathfrak{n} I=n+\left\langle\left(0, t_{2}^{4}\right),\left(0, t_{2}^{7}\right),\left(0, t_{2}^{10}\right)\right\rangle: \\
F_{12}\left(\lambda_{0}: \lambda_{1}\right)=\mathrm{S}\left\langle\left(\lambda_{0}, 1\right),\left(\lambda_{1}, t_{2}\right)\right\rangle, \\
F_{13}\left(\lambda_{0}: \lambda_{1}\right)=\mathrm{S}\left\langle\left(\lambda_{0}, 1\right),\left(\lambda_{1}, t_{2}\right),\left(0, t_{2}^{5}\right)\right\rangle, \\
I_{13}=\mathrm{S}\left\langle(0,1),\left(0, t_{2}\right),\left(1, t_{2}^{5}\right)\right\rangle, \\
I_{14}=\mathrm{S}\left\langle(1,0),(0,1),\left(0, t_{2}\right)\right\rangle . \\
\mathrm{S}^{\prime} I=\mathrm{D}_{1} \oplus \mathrm{B}^{\prime}, \mathfrak{n} I=n+\left\langle\left(0, t_{2}^{5}\right),\left(0, t_{2}^{7}\right),\left(0, t_{2}^{10}\right)\right\rangle: \\
I_{15}=\mathrm{S}\left\langle(1,1),\left(0, t_{2}^{2}\right),\left(0, t_{2}^{4}\right)\right\rangle \\
I_{16}=\mathrm{S}\left\langle(0,1),\left(1, t_{2}^{2}\right),\left(0, t_{2}^{4}\right)\right\rangle \\
I_{17}=\mathrm{S}\left\langle(0,1),\left(0, t_{2}^{2}\right),\left(1, t_{2}^{4}\right)\right\rangle . \\
\mathrm{S}^{\prime} I=\mathrm{R}_{0}: \quad \\
I_{18}=\mathrm{S}\left\langle(1,1),\left(0, t_{2}\right),\left(0, t_{2}^{2}\right)\right\rangle \\
I_{19}=\mathrm{S}\left\langle(0,1),\left(1, t_{2}\right),\left(0, t_{2}^{2}\right)\right\rangle \\
I_{20}=\mathrm{S}\left\langle(0,1),\left(0, t_{2}\right),\left(1, t_{2}^{2}\right)\right\rangle .
\end{aligned}
$$

Here $\mu$ denotes an element of $\mathbf{k}$ and $\left(\lambda_{0}: \lambda_{1}\right)$ a point of the projective line over $\mathbf{k}$.

Now pass to the calculation of R-ideals, which are not S-ideals. Again one can verify that for the following S-ideals $M$ we have $\mathfrak{m} M=\mathfrak{n} M$, so we do not need to consider the case, when $\mathrm{S} I=M$ :

$$
\begin{aligned}
F_{i}(\mu) & \text { for } i \neq 1,8 \\
F_{i}\left(\lambda_{0}: \lambda_{1}\right) & \text { for all } i, \\
F_{8}(\mu) & \text { for } \mu \neq 0, \\
I_{i} & \text { for } i \neq 2,7 .
\end{aligned}
$$

For $M=F_{1}(\mu), M / \mathfrak{m} M=\left\langle(1,1),\left(0, \mu t_{2}^{5}+t_{2}^{7}\right),\left(0, t_{2}^{8}\right)\right\rangle$. If $\mu \neq 0$, the multiplication by itself maps the second of these elements to the third one, which is congruent modulo $\mathfrak{m} M$ to $\mu^{-1} t_{2}^{10}$. Hence, here we obtain only the following new 1-parameter family of ideals:

$$
F_{14}(\mu)=\mathrm{R}\left\langle(1,1),\left(0, \mu t_{2}^{5}+t_{2}^{7}\right)\right\rangle, \mu \neq 0 .
$$

If $\mu=0$, we obtain the following new family:

$$
F_{15}(\mu)=\mathrm{R}\left\langle(1,1),\left(t_{2}^{7}+\mu t_{2}^{8}\right)\right\rangle .
$$

In the case $M=F_{8}(0), M / \mathfrak{m} M=\left\langle(1,1),\left(0, t_{2}^{4}+\mu t_{2}^{5}\right),\left(0, t_{2}^{8}\right)\right.$. Hence, we also obtain only one new family of ideals:

$$
F_{16}(\mu)=\mathrm{R}\left\langle(1,1),\left(t_{2}^{4}+\mu t_{2}^{5}\right)\right\rangle .
$$


The remaining cases: $\mathrm{S}$ and $I_{i}(i=2,7)$, give the ring $\mathrm{R}$ itself and the following ideals:

$$
\begin{aligned}
F_{17}\left(\lambda_{0}: \lambda_{1}\right) & =\mathrm{R}\left\langle(1,1),\left(\lambda_{0} t_{1}, \lambda_{1} t_{2}^{13}\right)\right\rangle, \\
F_{18}(\mu) & =\mathrm{R}\left\langle(1,1),\left(\mu t_{1}, t_{2}^{10}\right)\right\rangle, \\
I_{21} & =\mathrm{R}\langle(1,0),(0,1)\rangle \\
I_{22} & =\mathrm{R}\left\langle\left(1, t_{2}^{13}\right),(0,1)\right\rangle .
\end{aligned}
$$

Therefore, we have proved that in this case also $\operatorname{par}(1, R)=1$. Quite similar calculations prove the same for all other singularities of type $Z$.

Remark: Here the family $F_{13}$ is missing in Schappert's list of ideals.

\subsection{Ideals of singularities of type $E$}

Now consider the case of singularities $E$. In this case $d_{0}=3$, so it follows from [3] that each R-ideal is isomorphic either to an overring of $R$ or to its dual module. Therefore, we only need to find all overrings. But if $I$ is an overring of $\mathrm{R}$ then $\mathrm{SI}$ is also an overring of $\mathrm{S}$. Hence, at each stage of our inductive process we may restrict ourselves to overrings. To be complete, we always mark, which of these overrings are Gorenstein (i.e. self-dual).

Here we suppose that $s=3$ and $\mathrm{R}$ contains elements $x, y$ with $\mathbf{v}(x)=$ $(1,1,1)$ and $\mathbf{v}(y)=(\infty, k, 4)$ with $k>3$ (IUS of type $\left.\mathrm{E}_{2, p}\right)$. Of course, we suppose here that $x=t=\left(t_{1}, t_{2}, t_{3}\right)$. Again, passing to the minimal overring, we may suppose that $\mathrm{R}$ contains $\left(t_{1}^{k+2}, t_{2}^{k+2}, t_{3}^{5}\right) \mathrm{R}_{0}$ and is generated by $x$ and $y$ modulo this ideal. Then $\mathrm{S}$ contains $\left(t_{1}^{k+1}, t_{2}^{k+1}, t_{3}^{4}\right) \mathrm{R}_{0}$ and is generated modulo this ideal by $x$ and $z$, where $y=x z$ and $\mathbf{v}(z)=(\infty, k-1,2)$ (it is an IUS of type $\left.\mathrm{E}_{1, p}\right)$. The ring $\mathrm{S}^{\prime}$ contains $\left(t_{1}^{k}, t_{2}^{k}, t_{3}^{3}\right) \mathrm{R}_{0}$ and is generated modulo this ideal by $x$ and $z$. Put now $\mathfrak{n}^{\prime}=\operatorname{rad} \mathrm{S}^{\prime}, \mathrm{S}^{\prime \prime}=\operatorname{End}\left(\mathfrak{n}^{\prime}\right)$. Then $\mathrm{S}^{\prime \prime}$ is generated by $x$ and $z^{\prime}$, where $\mathbf{v}\left(z^{\prime}\right)=(\infty, k-2,1)$ and $z=x z^{\prime}$. Hence, $S^{\prime \prime}$ has only finitely many ideals up to isomorphism (it is a simple plane curve singularity of type D), cf. [6],[9]. Namely, here is the list of the overrings of $S^{\prime \prime}$ (except $\mathrm{S}^{\prime \prime}$ itself):

$$
\begin{aligned}
\mathrm{A}_{i} & =\mathrm{S}^{\prime \prime}\left\langle 1,\left(0, t_{2}^{i}, 0\right)\right\rangle \quad(1 \leq i \leq k-2), \\
\mathrm{B}_{i} & =\mathrm{S}^{\prime \prime}\left\langle 1,(0,0,1),\left(0, t_{2}^{i}, 0\right)\right\rangle \quad(0 \leq i \leq k-2), \\
\mathrm{B}_{01} & =\mathrm{S}^{\prime \prime}\langle 1,(1,0,0)\rangle, \\
\mathrm{B}_{02} & =\mathrm{S}^{\prime \prime}\langle 1,(0,1,0)\rangle, \\
\mathrm{R}_{0} & =\mathrm{S}^{\prime \prime}\langle(1,0,0),(0,1,0),(0,0,1)\rangle .
\end{aligned}
$$

Among them, only $\mathrm{A}_{i}$ are non-Gorenstein. Moreover, $M \mathfrak{n}^{\prime \prime}=M \mathfrak{n}^{\prime}$ for $M=$ $\mathrm{R}_{0}$ or $M=\mathrm{B}_{i}, i<k-2$, where $\mathfrak{n}^{\prime \prime}=\operatorname{rad} \mathrm{S}^{\prime \prime}$, so we do not need further 
to consider these overrings. Note also that in the case of $\mathrm{B}_{k-2}$ the generator $\left(0, t_{2}^{i}, 0\right)$ above is superfluous.

As $S$ is Gorenstein, its overrings, except $S$ itself, are those of $\mathrm{S}^{\prime}$. Here is the list of factoralgebas $M / \mathfrak{n}^{\prime} M$ for the overrings $M$ of $\mathrm{S}^{\prime \prime}$ :

$$
\begin{aligned}
S^{\prime \prime} / \mathfrak{n}^{\prime} & =\left\langle 1, z^{\prime},\left(0,0, t_{3}^{2}\right)\right\rangle \\
\mathrm{A}_{i} / \mathfrak{n}^{\prime} \mathrm{A}_{i} & =\left\langle 1,\left(0,0, t_{3}\right),\left(0, t_{2}^{i}, 0\right)\right\rangle, \\
\mathrm{B}_{k-2} \mathfrak{n}^{\prime} \mathrm{B}_{k-2} & =\left\langle 1,(0,0,1),\left(0, t_{2}^{k-2}, 0\right)\right\rangle, \\
\mathrm{B}_{01} / \mathfrak{n}^{\prime} \mathrm{B}_{01} & =\left\langle 1,(1,0,0),\left(0, t_{2}, 0\right)\right\rangle, \\
\mathrm{B}_{02} / \mathfrak{n}^{\prime} \mathrm{B}_{02} & =\left\langle 1,(0,1,0),\left(0, t_{2}, 0\right)\right\rangle .
\end{aligned}
$$

It is now easy to find all proper subalgebras of these algebras and, hence, the overrings of $S$ (except $S$ itself), which are not overrings of $S^{\prime \prime}$ :

$$
\begin{aligned}
\mathrm{S}^{\prime} & =\mathrm{S}\left\langle 1,\left(0,0, t_{3}^{3}\right)\right\rangle \\
\mathrm{A}_{k-1} & =\mathrm{S}\left\langle 1,\left(0,0, t_{3}^{2}\right)\right\rangle \\
F_{i}(\mu) & =\mathrm{S}\left\langle 1,\left(0, t_{2}^{i}, \mu t_{3}\right)\right\rangle \quad(1 \leq i \leq k-2) \\
\mathrm{B}_{k-1} & =\mathrm{S}\langle 1,(0,0,1)\rangle \\
\mathrm{B}_{01}^{\prime} & =\mathrm{S}\langle 1,(1,0,0)\rangle \\
\mathrm{B}_{02}^{\prime} & =\mathrm{S}\langle 1,(0,1,0)\rangle .
\end{aligned}
$$

Among them only $\mathrm{S}^{\prime}, \mathrm{A}_{k-1}$ and $F_{i}(0)$ are non-Gorenstein. Note also that $M \mathfrak{m}=M \mathfrak{n}^{\prime}$ for all overrings of $\mathrm{S}^{\prime \prime}$, so we do not need to consider them further. To find all overrings of $\mathrm{R}$, which are not overrings of $\mathrm{S}$, calculate the factoralgebras $M / \mathfrak{m} M$ for the rings of the preceding list and $\mathrm{S}$ :

$$
\begin{aligned}
\mathrm{S} / \mathfrak{m} & =\left\langle 1, z,\left(0,0, t_{3}^{4}\right)\right\rangle, \\
\mathrm{S}^{\prime} / \mathfrak{m S}^{\prime} & =\left\langle 1, z,\left(0,0, t_{3}^{3}\right)\right\rangle, \\
\mathrm{A}_{k-1} / \mathfrak{m A}_{k-1} & =\left\langle 1,\left(0,0, t_{3}^{2}\right),\left(0,0, t_{2}^{k-1}\right)\right\rangle, \\
F_{i}(\mu) / \mathfrak{m} F_{i}(\mu) & =\left\langle 1,\left(0, t_{2}^{i}, \mu t_{3}\right),\left(0,0, t_{3}^{2}\right)\right\rangle, \\
\mathrm{B}_{k-1} / \mathfrak{m B}_{k-1} & =\left\langle 1,(0,0,1),\left(0, t_{2}^{k-1}\right)\right\rangle, \\
\mathrm{B}_{01}^{\prime} / \mathfrak{m B}_{01}^{\prime} & =\left\langle 1,(1,0,0),\left(0, t_{2}^{2}, 0\right)\right\rangle, \\
\mathrm{B}_{02}^{\prime} / \mathfrak{m B}_{02}^{\prime} & =\left\langle 1,(0,1,0),\left(t_{1}^{2}, 0,0\right)\right\rangle .
\end{aligned}
$$

It gives us the following list of overrings of $R$, which are not overrings of $S$ 
(except $\mathrm{R}$ itself):

$$
\begin{aligned}
\mathrm{R}^{\prime} & =\mathrm{R}\left\langle 1,\left(0,0, t_{3}^{4}\right)\right\rangle, \\
F_{k-1}^{\prime}(\mu) & =\mathrm{R}\left\langle 1, \mu z+\left(0,0, t_{3}^{3}\right)\right\rangle, \\
F_{k-1}(\mu) & =\mathrm{R}\left\langle 1,\left(0, \mu t_{2}^{k-1}, t_{3}^{2}\right)\right\rangle \quad(\mu \neq 1), \\
F_{i}^{\prime}(\mu) & =\mathrm{R}\left\langle 1,\left(0, t_{2}^{i}, t_{3}^{2}\right)\right\rangle \quad(1 \leq i \leq k-2), \\
\mathrm{B}_{k} & =\mathrm{R}\langle 1,(0,0,1)\rangle, \\
\mathrm{B}_{01}^{\prime \prime} & =\mathrm{R}\langle 1,(1,0,0)\rangle, \\
\mathrm{B}_{02}^{\prime \prime} & =\mathrm{R}\langle 1,(0,1,0)\rangle .
\end{aligned}
$$

Here $F_{k-1}(\mu), \mathrm{B}_{k}, \mathrm{~B}_{01}^{\prime \prime}$ and $\mathrm{B}_{02}^{\prime \prime}$ are Gorenstein.

Thus, we have proved that $\operatorname{par}(1, R)=1$. Analogous calculations show the same for all other IUS of type $\mathrm{E}$, which accomplishes the proof of Theorem 2.1.

\section{References}

[1] Arnold V.I., Varchenko A.N., Gusein-Zade S.M., Singularities of Differentiable Maps, Vol. 1, Birkhäus er, Boston-Basel-Stuttgart, 1985.

[2] Bass H., On the ubiquity of Gorenstein rings, Math. Z. 82 (1963), 8 - 28.

[3] Drozd Yu.A., Ideals of commutative rings, Mat. Sbornik, 101 (1976) 334348.

[4] Drozd Yu.A., Greuel G.-M., Cohen-Macaulay module type, Compositio Math. 89 (1993) 315-338.

[5] Drozd Yu.A., Greuel G.-M., Semicontinuity for representations of CohenMacaulay rings, Preprint Nr. 247, Fachbereich Math. Univ. Kaiserslautern, 1993. To appear in Math. Ann. 1996

[6] Drozd Yu.A., Roiter A. V., Commutative rings with a finite number of indecomposable integral representations Izv. Aad. Nauk SSSR. Ser. Mat. 31 (1967) 783-798.

[7] Greuel G.-M., Knörrer H., Einfache Kurvensingularitäten und torsionfreie Moduln, Math. Ann. 270 (1985) 417-425.

[8] Greuel G.-M.; Kröning H., Simple singularities in positive characteristic, Math. Z. 203, (1990) 339-354.

[9] Jacobinski H., Anneaux commutatifs avec un nombre fini de reseaux indecomposable, Acta Math. 118 (1967) 1-31. 
[10] Knörrer H., Torsionfreie Moduln bei Deformation von Kurvensingularitäten, In: Greuel G.-M., Trautmann G. (ed.) Singularties, Representations of Algebras and Vector Bundles, Lambrecht 1985. Lecture Notes in Math., Vol. 1273, Springer, Berlin-Heidelberg-New York (1987) 150-155.

[11] Schappert A., A characterization of strict unmodal plane cure singularities, In: Greuel G.-M., Trautmann G. (ed.) Singularties, Representations of Algebras and Vector Bundles, Lambrecht 1985. Lecture Notes in Math., Vol. 1273, Springer, Berlin-Heidelberg-New York (1987) 168-177.

[12] Schappert A., Kurvensingularitäten und Isomorphieklassen von Moduln, Dissertation, Universität Kaiserslautern, 1990.

[13] Wall C.T.C., Classification of unimodal isolated singularities of complete intersections, In: Orlik P. (ed.) Singuarities, Arcata 1981. Proc. Sympos. Pure Math. 40(2) (1983) 625-640. 